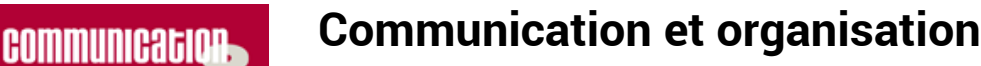

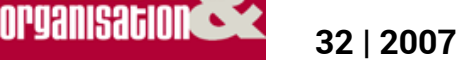

La ville dans tous les sens

\title{
La polyphonie urbaine : essai de définition
}

Bernard Lamizet

\section{OpenEdition}

Journals

Édition électronique

URL : http://journals.openedition.org/communicationorganisation/1141

DOI : 10.4000/communicationorganisation. 1141

ISSN : $1775-3546$

\section{Éditeur}

Presses universitaires de Bordeaux

\section{Édition imprimée}

Date de publication : 1 décembre 2007

Pagination : 14-25

ISSN : 1168-5549

\section{Référence électronique}

Bernard Lamizet, «La polyphonie urbaine : essai de définition », Communication et organisation [En ligne], 32 | 2007, mis en ligne le 01 décembre 2010, consulté le 10 décembre 2020. URL : http:// journals.openedition.org/communicationorganisation/1141 ; DOI : https://doi.org/10.4000/ communicationorganisation. 1141 
Dossier : La ville dans tous les sens

\section{Résumé}

Dans la ville, se mêlent les appartenances, les sociabilités, les cultures, les identités, se confrontent et se mêlent les différences et les antagonismes. La communication urbaine est donc une polyphonie, qui s'exprime dans les formes conflictuelles de la communication politique et dans les formes intégratrices de la médiation culturelle. Il s'agit d'une forme sémiotique de communication inscrivant les formes de l'expression et les dynamiques de la signification et de l'interprétation dans des lieux de communication qui articulent dans l'esthétique et la culture l'identité des acteurs et leurs langages dans la géographie politique de l'espace public. L'article envisage aussi les ruptures de la communication urbaine et les silences de la ville.

\section{Mots-clés}

Culture, esthétique, identité, médiation, sémiotique urbaine

\section{Abstract}

In towns, identities, cultures, social links are confrontated to each other. Therefore, urban communication is a polyphony expressed in conflictual forms of political languages and in integrative forms of cultural mediation. That semiotic system is present in a political geography of places of communication with esthetical and cultural identities. The paper pays also attention to silences and breakings of urban communication.

\section{Key-Words}

Aesthetics, culture, identity, mediation, urban semiotics

\section{Bernard Lamizet}

Bernard Lamizet est Professeur en Sciences de l'Information et de la Communication à l'Institut d'Etudes Politiques de Lyon. Il est chercheur dans l'équipe ELICO, Equipe lyonnaise en sciences de l'Information et de la Communication, commune à l'ensemble des universités lyonnaise. 


\section{La polyphonie urbaine : essai de définition Bernard Lamizet}

bernard.lamizet@univ-lyon2.fr

Pour reprendre les travaux de M. Bakhtine ${ }^{1}$, un espace polyphonique est un espace qui met en scène ensemble plusieurs langages et plusieurs codes distincts les uns des autres, plusieurs systèmes symboliques différents d'expression et de représentation. Mais, surtout, un espace polyphonique n'est pas un espace de bruits et de confusion sonore : il s'agit d'un espace dans lequel l'articulation entre ces codes et ces langages divers s'inscrit dans une logique esthétique, en ce que, d'une part, elle répond à des exigences esthétiques, et, d'autre part, elle élabore une signification complexe.

La ville se fonde, précisément, sur l'existence et la rencontre de plusieurs codes différents et de plusieurs systèmes distincts d'expression et de signification. La culture urbaine est caractérisée par le fait qu'elle s'élabore dans un espace d'indistinction où se mêlent les appartenances, les sociabilités, les cultures, les identités, dans une logique de confrontation et de métissage des différences et des antagonismes. Ce qui fait naître la ville, à la fois comme espace politique et comme espace culturel, c'est la rencontre, le rapport à la différence et à la multiplicité des codes et des voies. La ville est un espace qui naît d'une rencontre car elle se fonde sur le carrefour qui fait se croiser plusieurs parcours et plusieurs histoires, plusieurs identités et plusieurs projets, plusieurs engagements et plusieurs appartenances. La communication qui se déploie dans l'espace urbain consiste, dans ces conditions, dans une véritable polyphonie que l'on peut définir par quatre éléments majeurs.

D'une part, cette communication met en œuvre des langages et des formes multiples d'expression et de signification. L'espace urbain met en scène une confrontation incessante entre des langages et des cultures qui se rencontrent au cours de l'histoire de la ville. C'est ainsi, par exemple, qu'une ville comme Marseille a vu se succéder et se rencontrer, dans son site, la culture grecque, la culture française, des cultures orientales, la culture italienne, et d'autres identités qui ont $\mathrm{pu}$ inscrire leurs traces et leurs représentations dans l'espace de la ville. Une telle succession de cultures différentes s'exprime, en

\footnotetext{
${ }^{1}$ Bakhtine introduit la notion de polyphonie dans ses travaux sur l'esthétique du roman et la littérature, présentés, en particulier, par J. Kristeva (Cf. Kristeva (1969), p. 143-173.
} 
particulier, par les noms de lieux (le Pharo, emplacement d'une tour grecque, la Canebière, trace du travail du chanvre) et par les objets du patrimoine (le quartier de la Bourse révèle une partie des vestiges de la cité grecque). D'autre part, la communication urbaine comporte une part considérable d'imprévisible et d'aléatoire. La ville est un espace dans lequel surviennent des événements qui ne sont pas toujours prévisibles, comme peuvent l'être les événements naturels. C'est en raison de cette part d'aléatoire considérable de la culture urbaine que sont nés, d'une part, la presse comme système d'information et de mémorisation sur l'imprévu, et, d'autre part, le politique, comme ensemble des systèmes sociaux d'attente et de réponse institutionnelle à l'imprévu et à l'accidentel (institutions de régulation, police, journaux qui veillent sur l'inattendu et informent la population quand il survient).

Par ailleurs, la polyphonie urbaine s'exprime à la fois dans les formes conflictuelles de la communication politique et dans les formes intégratrices de la médiation culturelle. Dans l'espace de la ville, s'articulent les unes aux autres, d'une part des logiques sociales et institutionnelles d'intégration et, d'autre part, des pratiques culturelles et esthétiques de mise en scène de l'étranger et de l'associable. D'une part, la politique de la ville, comme, par exemple, les systèmes politiques de prévention sociale, ou la politique de l'école, vise à l'intégration de toutes les populations dans une logique unificatrice de l'identité urbaine. C'est aussi pourquoi l'espace urbain a aussi connu les premières formes de stratégies de maintien de l'ordre public. D'autre part, l'espace urbain met en scène des cultures différentes et étrangères les unes aux autres, dans une logique de représentation et de confrontation de ces cultures les unes aux autres. C'est pourquoi, par exemple, la ville est un espace de manifestations politiques au cours desquelles les identités politiques expriment leur antagonisme.

Enfin, la polyphonie urbaine désigne une sémiotique de la communication qui articule les formes de l'expression et les dynamiques de la signification et de l'interprétation. L'espace urbain est un espace dans lequel se lisent et se déchiffrent les signes de la culture et de la sociabilité ${ }^{2}$. Dans l'espace urbain, les pratiques sociales ne se donnent pas seulement à voir, mais se donnent aussi à lire ; dans la ville, il ne faut pas seulement découvrir les pratiques des habitants et des visiteurs, mais aussi comprendre qu'il s'agit de logiques sociales porteuses de significations à comprendre et à interpréter.

\footnotetext{
${ }^{2}$ Cf. Paquot (1990).
} 


\section{Géographie symbolique de l'espace urbain}

Une approche sémiotique de l'espace de la ville pense un espace urbain structuré par des signes. Lieux de la rencontre et de l'échange, les lieux de la ville sont des lieux de communication qui articulent l'identité qu'ils expriment et les langages qui y sont mis en œuvre par les acteurs de la communication et de la signification ${ }^{3}$. En effet, dans la ville, on est toujours confronté à l'identité de l'autre, par rapport à laquelle on structure sa propre identité ${ }^{4}$. La différence majeure entre l'espace naturel et l'espace urbain réside peut-être, justement, dans cette différence entre les deux modes de structuration qui les instituent. L'espace de la nature est structuré par des lieux, des sites et des espaces qui, comme les montagnes, les fleuves ou les littoraux, résultent d'événements et de processus qui atteignent la configuration des sites sans l'intervention des hommes, des cultures et des sociétés. En revanche, l'espace de la ville est, lui, structuré par des repères et des aménagements, par des informations et des orientations, institués par les systèmes sociaux qui régulent et organisent la vie urbaine. Dans la ville, même les configurations liées à la nature sont socialisées par les institutions urbaines : les fleuves qui traversent les villes sont encadrés par des berges aménagées ${ }^{5}$ et enjambées par des ponts, les mers qui bordent les espaces urbains sont aménagées par des ports et par des quais. Le rôle de la voirie est, ainsi, de socialiser, d'institutionnaliser, les usages de l'espace urbain. Tous ces aménagements sont autant de supports de signes et de codes. On peut faire apparaître, dans l'espace de la ville, cinq grands lieux de communication et d'expression, qui, ensemble, dessinent une géographie symbolique de l'espace urbain.

Les lieux de la circulation et du déplacement font apparaître une communication mobile, nomade. La communication urbaine est une communication du déplacement et de la rencontre. C'est ainsi, d'une part, que l'on découvre les signes de la culture urbaine en se déplaçant le long des rues, en lisant leurs noms et leur signalisation et en lisant les affiches et les enseignes, et, d'autre part, que l'on peut apprécier aussi la polyphonie urbaine par la variété des signes portés et exprimés par les acteurs qui parcourent l'espace urbain. Par exemple, véhicules de transports en commun et véhicules utilitaires portent des

\footnotetext{
${ }^{3}$ Cf. Lamizet (2002) et Roncayolo (1997).

${ }^{4}$ Cf. les recherches de Lacan sur l'identité et le «stade du miroir». Cf. aussi Laplantine (1999).

${ }^{5}$ C'est ainsi, par exemple, qu'à Lyon, les quais du Rhône viennent d'être aménagés, du nord au sud de l'espace urbain, par l'installation d'un Parc des berges du Rhône.
} 
informations et des signes et leur font parcourir les rues. Publicité et indications de destination rendent, ainsi, l'espace urbain lisible.

La polyphonie urbaine articule aussi systèmes collectifs d'information et de communication et systèmes privés et singuliers d'expression de l'identité. Les lieux de l'habitation sont les lieux de l'ancrage identitaire dans un espace propre, ceux de la communication privée et ceux de l'intersubjectivité. La différenciation entre espaces privés et espace public constitue, ainsi, une forme de plus de l'élaboration de l'espace urbain en un système de signes. C'est une autre logique d'information que l'on peut lire dans la ville, en découvrant, par les portes et par les fenêtres, les aménagements des espaces privés. L'espace urbain met en scène toute une sémiotique du singulier à travers aussi les postures et les vêtements des habitants et des visiteurs qui le parcourent, mais aussi à travers les pratiques de communication singulière mises en œuvre dans l'espace urbain : lecture des journaux, communications téléphoniques grâce aux téléphones portables, conversations qui ont lieu au fil des rencontres, viennent aussi nourrir la polyphonie de l'espace urbain et accentuer son imprévisibilité.

Les lieux de l'échange et du commerce sont les lieux de la communication publicitaire et de la négociation. Il s'agit d'un autre espace de la polyphonie urbaine, qui repose, lui, sur ce que l'on peut appeler une polyphonie marchande. Le marché urbain a toujours été une polyphonie, faite des cris des marchands dans les marchés, des vitrines et des affiches publicitaires dans les rues, des informations communiquées dans les lieux institués de l'échange et du commerce comme les bourses, les banques et les entreprises de transports. Villon, au Moyen Âge, et Boileau au XVII ${ }^{\mathrm{e}}$ siècle, ont illustré, dans la littérature cette forme sonore de la polyphonie urbaine.

Les agoras sont les lieux du débat politique et de la confrontation. La ville est l'espace du politique, car elle est l'espace du débat public, des pouvoirs et de la représentation. En ce sens, la polyphonie urbaine comporte une dimension proprement politique, qui est, elle-même, une polyphonie, car elle est un système de communication dans lequel les identités politiques différentes s'opposent les unes aux autres dans la confrontation permanente du débat et des pratiques politiques. La polyphonie politique de l'espace urbain s'exprime, en particulier, dans les défilés et les manifestations de protestation, dans la confrontation des partis et des journaux engagés et dans la mise en scène du débat institutionnel dans les parlements et dans les lieux du vote et de la délibération. La rue devient, ainsi, un lieu de polyphonie politique quand elle est parcourue par des manifestants qui font d'elle un espace 
de confrontation politique.

Enfin, l'espace urbain connaît les lieux de la communication de crise, ou de la communication de souffrance, que sont les hôpitaux et les lieux de la relégation urbaine. La polyphonie urbaine est ici celle de la santé et de la maladie, celle du bien-être et de la misère, celle de l'ordre et de la souffrance. L'espace urbain a toujours connu une coexistence, une confrontation, souvent conflictuelle, entre deux façons d'habiter la ville : celle qui l'habite en se l'appropriant et en y trouvant ses marques, et celle qui l'habite en s'en faisant rejeter, en vivant une véritable exclusion de l'espace urbain. La cour des miracles a toujours été une caractéristique de l'espace urbain au même titre que les lieux du pouvoir. Les hôtels, les hôpitaux, les lieux d'accueil de la précarité sont, ainsi, des lieux caractéristiques de la sociabilité urbaine ${ }^{6}$.

\section{Politique et communication urbaine}

Le propre de la communication mise en œuvre dans l'espace urbain est d'être politique, en quelque sorte par nature et par définition. En effet, elle est élaborée, exprimée et interprétée par des acteurs qui, par leur pratique même de la communication, expriment des identités politiques. Sans doute est-ce la raison pour laquelle la communication urbaine est nécessairement une polyphonie. C'est que cette communication ne met pas en scène des pratiques symboliques mises en œuvre par des acteurs porteurs d'une identité unique, mais des pratiques symboliques dont les auteurs et les énonciateurs expriment des identités différentes et antagonistes. Les « voix » qui composent la polyphonie urbaine sont celles d'acteurs différents les uns des autres, porteurs de cultures et de savoirs étrangers les uns aux autres, énonçant des messages renvoyant à des réalités distinctes. C'est ce qui explique que la communication urbaine soit, en un sens, nécessairement politique, car elle consiste toujours à donner du sens à cette confrontation d'une multiplicité d'identités différentes.

La communication urbaine, dans ces conditions, fait apparaître une polyphonie faite d'abord de la confrontation entre les discours du pouvoir et les discours des oppositions. Les institutions urbaines sont chargées d'une polyphonie qui leur est propre, qui consiste dans le dialogue, parfois violent, entre les pouvoirs de la ville et les acteurs politiques qui les contestent ou s'opposent à eux, dans les lieux de la délibération, dans l'affichage et dans les médias urbains. L'affichage,

\footnotetext{
${ }^{6}$ Cf. « Vivre à l'hôtel », dans Le Monde, 4 nov. 2007. Cf. aussi Marec (2005), p. 429 (sur Pasteur) et p. 665 (sur Bologne) et Hoggart (1970).
} 
par exemple, exprime sur les murs cette polyphonie, ainsi faite de dimensions différentes ( $4 \mathrm{~m} \mathrm{x} 3 \mathrm{~m}$, mais aussi d'autres dimensions), et de thématiques différentes (politiques et commerciales). La polyphonie urbaine oppose ensuite les discours des institutions et les discours de l'errance ${ }^{7}$. La ville a, depuis toujours, mis en scène deux langues, deux cultures, celle, d'un côté, de la culture établie, légitime, et, celle, de l'autre, de la pauvreté, de l'argot et de l'exclusion. Cette polyphonie particulière donne à la communication urbaine la spécificité d'une véritable opposition entre des langues qui ne se comprennent pas. Par ailleurs, dans l'espace de la ville, les discours du projet s'opposent à ceux de la mémoire. Cette polyphonie, proprement politique, oppose les uns aux autres les langages de la modernité et ceux de la tradition, dans des systèmes architecturaux différents, mais aussi dans des discours esthétiques et politiques différents sur l'espace urbain $^{8}$. Enfin, l'espace de la ville est le cadre d'une confrontation entre les discours de la croyance et les discours de la critique et de la contestation. La ville a toujours connu la cohabitation, souvent conflictuelle elle aussi, entre les lieux du culte et ceux de la libre pensée, entre les formes religieuses d'expression et d'identification et les formes laïques de l'engagement politique. C'est ainsi qu'à Lyon, la fin du XIX ${ }^{\mathrm{e}}$ siècle a connu une opposition forte entre les canuts, les ouvriers de la soie, et les institutions urbaines, et entre les mouvements laïcs de gauche et les institutions religieuses (fête de l'Immaculée Conception le 8 décembre).

Tous ces discours et toutes ces formes de la communication constituent ensemble l'espace public, système spatial de représentation et d'expression des identités politiques. C'est J. Habermas, en $1962^{9}$, qui élabore ce concept nouveau d'espace public, appelé à exprimer et à représenter toutes les formes, symboliques et politiques, que peut revêtir le débat public dans les différents événements et les différentes situations dans lesquels il est mis en œuvre par les acteurs politiques de l'espace urbain. Propre, sans doute, à la ville, cet espace de rencontre et de circulation, qui, dans une culture démocratique, ne saurait faire l'objet d'aucune appropriation, est l'espace, commun à tous, dans lequel peuvent s'exprimer et s'échanger librement les opinions et les engagements qui marquent les identités politiques présentes dans l'espace de la ville.

\footnotetext{
${ }^{7}$ Voir, sur ce sujet, Declerck (2001) et Hoggart (1970).

${ }^{8}$ Voir, en particulier, sur ce point, l'œuvre de Balzac, celle de Dickens ou celle de V. Hugo.

${ }^{9}$ Cf. Habermas (1993).
} 


\section{Médiation esthétique et culturelle de l'urbanité}

La polyphonie urbaine donne lieu à une esthétique particulière, qui articule la représentation et l'expression des identités sociales et politiques et celle d'idéaux culturels, exprimés par l'art et par l'esthétique. C'est parce qu'elle s'inscrit dans une logique de médiation esthétique que la multiplicité des codes de la ville est bien une polyphonie, et non une cacophonie. Il ne s'agit pas de bruits qui se succèderaient les uns aux autres et se brouilleraient les uns les autres dans une multiplicité sans logique. En revanche, il s'agit bien de langages qui s'inscrivent, de façon dialogique et complémentaire, dans un système sémiotique commun.

La médiation culturelle de l'urbanité s'inscrit dans l'espace de la ville qui constitue, dans ces conditions, un espace de mise en valeur et de représentation des formes esthétiques de son expression et de ses langages. La polyphonie urbaine est une médiation, en ce qu'elle institue une dialectique entre le singulier et le collectif : chacune des voix qui participent à la polyphonie urbaine a son identité propre, distincte et reconnaissable, mais, dans le même temps, l'ensemble de ces voix se retrouvent dans un projet collectif, dans un système collectif qui exprime une identité d'ensemble. Mais elle est une médiation esthétique, en ce que le projet commun qui donne sa signification à l'ensemble des voix exprimées est un projet consistant dans une matérialité perceptible qui s'adresse aux sens autant qu'à l'interprétation.

On peut, en particulier, analyser, ici, trois types de médiation esthétique et culturelle de l'urbanité qui s'expriment dans des lieux et dans des langages propres. La monumentalité met en scène une polyphonie visible. Les monuments donnent à voir la référence aux événements et aux personnages dont ils expriment la mémoire. La ville est un espace de constructions, un espace structuré, dans ces conditions, par de l'architecture ${ }^{10}$. Les constructions qui jalonnent l'espace urbain y inscrivent une polyphonie de styles architecturaux différents, d'époques différentes de construction, de destinations et de significations différentes des constructions et des paysages urbains. C'est en parcourant la ville du regard que l'on peut, ainsi, prendre conscience des structures de cette polyphonie, et que l'on peut aménager une polyphonie du paysage urbain dans laquelle on peut trouver des significations et des formes esthétiques. La monumentalité est une polyphonie qui s'adresse au regard et à une culture visuelle de

\footnotetext{
${ }^{10}$ Cf. Ragon (1991).
} 
la ville.

Le spectacle met en scène une polyphonie audible et visible. La ville a toujours été un espace dans lequel étaient organisés et mis en scène des spectacles destinés au peuple assemblé. L'espace public, les rues et les places, puis, dans une clôture de l'esthétique des spectacles, les théâtres, les cinémas et les salles de concert, a toujours offert à ses habitants des spectacles associant la dimension visible de la polyphonie urbaine, par les acteurs et les scénographies, et sa dimension audible, par les musiques, les danses et les déclamations. Les travaux de J. Vilar, mais, plus récemment, ceux de J. Caune et de P. Chaudoir ont bien mis en évidence cette dimension essentielle de la polyphonie urbaine ${ }^{11}$. L'écriture met en scène une polyphonie lisible. C'est que l'espace urbain est un espace de signes qui se donnent à lire autant qu'à voir. On a évoqué plus haut l'indication du nom des rues et des sites de la ville. Mais il faut, ici, évoquer tous ces signes visibles dont la ville offre une polyphonie active. Des affiches aux enseignes lumineuses, des devantures ${ }^{12}$ aux étals des marchands ambulants, des informations aux "petites annonces » collées sur les murs et les panneaux de signalisation, la ville offre au regard une quantité considérable d'informations et de discours lisibles distincts, énoncés dans des langues différentes et dans des systèmes symboliques variés.

\section{Les silences de la ville et les ruptures de la communication urbaine}

Mais, comme tout système de communication, la ville connaît ses silences, ses ruptures, ses obstacles à la communication. Sans doute est-ce même aussi la raison pour laquelle il s'agit d'une polyphonie. En effet, on se rend compte de la dimension d'ensemble d'une polyphonie précisément quand certains sons ou certaines voix s'expriment de façon différente des autres, créant, ainsi, une sorte de «crise sonore». La polyphonie ne s'identifie comme telle qu'en s'opposant à la cacophonie. Ces crises et ces fractures de la communication urbaine peuvent tenir à cinq types de facteurs.

Des événements peuvent se produire dans l'espace urbain, qui le plongent dans le silence. Dans certaines situations, la ville ne peut plus s'exprimer, elle ne peut plus se parler à elle-même. C'est, en particulier, le cas lors de catastrophes «naturelles», quand, au

\footnotetext{
${ }^{11} \mathrm{Cf}$. Les travaux d'habilitation à diriger des recherches de P. Chaudoir (Université Lyon II, 2006), Vilar (1966) et Caune (1999).

${ }^{12} \mathrm{Cf}$. Demestreco et Martins (2007).
} 
vacarme assourdissant des bruits de la violence de la nature, succèdent l'interrogation des habitants et le silence des sauveteurs qui recherchent les victimes sous les décombres. Des conflits et des antagonismes peuvent donner lieu à une violence de nature à paralyser l'exercice de la communication. La guerre fait partie de ces situations de violence dans laquelle la polyphonie urbaine ne s'entend plus, parce que ses sonorités et ses langages sont recouverts par le bruit des affrontements et des violences qui font perdre la conscience des identités urbaines. C'est ainsi qu'en novembre 2002, par exemple, la polyphonie des manifestations et des conflits sociaux a pleinement habité l'espace urbain et lui ont donné une signification particulière.

Certaines mutations de la sociabilité urbaine et certaines transformations de l'espace urbain, mal assumées ou mal comprises par les habitants de la ville, peuvent donner lieu à des temps d'incommunicabilité. C'est ce qui peut se produire lors de la rénovation imposée de certains quartiers, ou lorsque certains quartiers ou certains sites urbains perdent leurs habitants. Dans ces situations, la polyphonie urbaine devient, en fait, la polyphonie conflictuelle des anciens sons et des anciennes voix qui s'opposent aux nouveaux, qui ne peuvent s'imposer pleinement car ils ne sont pas reconnus par les habitants. A Lyon, les langages nouveaux de la Croix-Rousse ont du mal à coexister, ainsi, avec les anciens ${ }^{13}$. Des conflits et des tensions peuvent être liés à des projets architecturaux et à des mutations esthétiques de l'espace urbain. La polyphonie visuelle de la ville peut, ainsi, être heurtée par la coexistence de langages et de styles architecturaux opposés les uns aux autres. C'est ce qui a pu se produire, par exemple, quand le quartier de la Part-Dieu a imposé, à Lyon, le langage d'une modernité offensive dans un quartier urbain qui se pensait dans une autre logique. Enfin, des crises de l'économie urbaine et des identités sociales peuvent entraîner des ruptures des médiations de l'urbanité. La mutation, à Lyon, du quartier de la CroixRousse, du tissage traditionnel des canuts à la modernité urbaine de la culture et des spectacles, mais aussi, la mutation, à Paris, des sons du quartier Marais a fait changer ce quartier de sonorités et de langage. Une polyphonie s'y installe entre les langages de plusieurs temporalités.

\section{Conclusion : la sémiotique urbaine}

C'est le rôle de la sémiotique urbaine de proposer les outils d'intelligibilité et d'interprétation de ces polyphonies urbaines, y

\footnotetext{
${ }^{13}$ Cf. Roncayolo (2001), p. 503 et 669.
} 
compris de leurs silences et de leurs ruptures. En rendant intelligible la diversité des sons de la ville, le but de la sémiotique urbaine est d'inscrire dans l'espace urbain la polyphonie des médiations culturelles et des médiations politiques. On peut, en particulier, souligner quatre éléments méthodologiques et épistémologiques majeurs de ce projet sémiotique. Le premier élément important est de marquer, ainsi, la vocation des sciences de l'Information et de la Communication, qui est de concourir à l'élaboration d'une rationalité des formes urbaines de la communication et de l'analyse politique. Si les sciences de l'Information et de la Communication sont nées dans l'espace urbain, il est essentiel de les penser, aujourd'hui comme un mode d'approche et de rationalité spécifique de la ville, voire comme un mode d'intelligibilité du fait urbain susceptible de contribuer de façon spécifique à la résolution des crises de la ville. Le second élément sur lequel nous souhaitons insister en conclusion est le fait que la polyphonie urbaine est, sans doute, une expression spécifique de la politique de la ville. D'une part, la polyphonie urbaine est fondamentalement politique, car elle fait apparaître la spécificité et la différence des acteurs institutionnels qui prennent part à la communication urbaine. D'autre part, la polyphonie urbaine inscrit dans l'histoire de la ville les conflits et les antagonismes qui s'expriment dans l'espace urbain. Le troisième élément important de cette recherche est la nécessité de penser la ville comme un espace de langages et de représentations. La dimension proprement et nécessairement sémiotique de l'espace urbain est ce qui le distingue fondamentalement d'autres espaces. La ville est un espace qui, en quelque sorte, n'existe que dans une perspective sémiotique, dans la dimension symbolique d'un système de signes interprétables. Enfin, sans doute est-il important de souligner que, dans la ville, comme dans tout système sémiotique, il existe une part essentielle d'inconscient, de refoulé, de caché. Les sciences de l'Information et de la Communication, et, en particulier, la sémiotique urbaine, ont, justement, à faire apparaître les logiques et les structures de cet inconscient de la ville et de leur donner du sens.

\section{Bibliographie}

Calvino I., Les villes invisibles, tr. fr. par J. Thibaudeau, Paris, Editions du Seuil, 1996, 193 p. (Coll. « Points »), 1972.

Caune J., Pour une éthique de la médiation. Le sens des pratiques culturelles, Grenoble, Editions Presses Universitaires de Grenoble, 294 p. (Coll. « Communication, médias et sociétés »), 1999. 
Declerck P., Les naufragés, Paris, Editions Plon, 458 p., bibl., ind. (Coll. « Terre humaine »), 2001.

Demetresco S. et Martins M., Vitrines : entre la tentation et la séduction, Communication présentée au congrès de l'A.I.S (Lyon, 2004) : http://jgalith.univ-lyon2.fr/Actes, 2007.

Habermas J., L'espace public (1962), tr. par M. B. de Launay, Paris, Payot, 324 p. (Coll. « Critique de la politique »), 1993.

Hoggart R., La culture du pauvre, tr. fr. par F. et J.-C. Garcias et J.C. Passeron, Paris, Éditions de Minuit, 420 p., bibl., ind. (Coll. « Le sens commun »), 1970.

Kristeva J., Séméiotikè, Paris, Editions du Seuil, 381 p ; (ind.) (Coll. « Tel Quel »), 1969.

Lamizet B. et Sanson P. et al., Les langages de la ville, Marseille, Editions Parenthèses, 187 p. (Coll. « Eupalinos »), 1997.

Lamizet B., le sens de la ville, Paris, Editions L'Harmattan, 242 p. (Coll. « Villes et sociétés ») 2002.

Laplantine F., Je, nous et les autres, Paris, Editions du PommierFayard, 156 p. (Coll. « Manifestes »), 1999.

Marec Y., (sous la dir. de), Villes en crise ?, Paris, Edition Creaphis, 2005.

Paquot T., Homo urbanus, Paris, Editions du Félin, 1990.

Ragon M., Histoire de l'architecture et de l'urbanisme moderne (1986), Paris, Edition du Seuil, 1991.

Roncayolo M., (sous la dir. de), La ville aujourd'hui, Paris, Editions du Seuil, 903 p. (Coll. « Points »), 2001.

Roncayolo M., La ville et ses territoires, Paris, Editions Gallimard, 285 p., bibl. (Coll. « Folio »), 1997.

Vilar J., De la tradition théâtrale, Paris, Gallimard, 189 p. 1966.

Revue Française de Science politique, « La ville objet politique, objet de politiques» Paris, Presses de Sciences Po, vol. 45, $\mathrm{n}^{\circ} 1$, févr. 1995. 